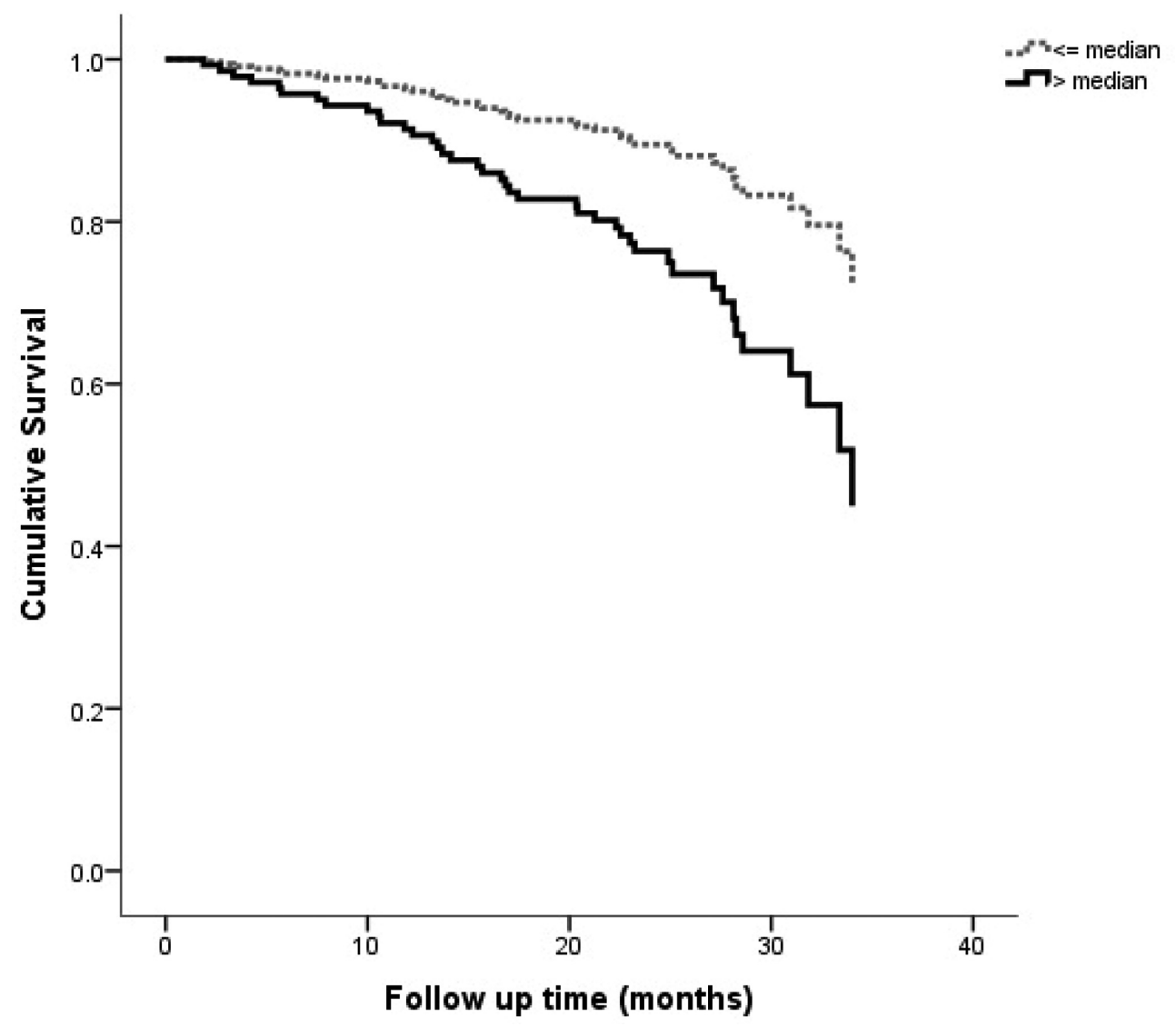

Abstract 128 Figure 1 Survival in $<=$ median versus $>$ median GLS (median $=-13.7 \%$ )

mortality in stable HD patients than the standard echocardiographic parameters LVEF and $\mathrm{LVMIHt}^{2.7}$.

\section{IMPACT OF CARDIOVASCULAR MAGNETIC RESONANCE ON MANAGEMENT AND CLINICAL DECISION-MAKING IN ACUTE HOSPITALISED PATIENTS}

Estefania De Garate*, Amardeep Ghosh Dastidar, Anna Baritussio, Alessandra Scatteia, Antonio Amadu, Giuseppe Venuti, Jonathan C Rodrigues, Chiara Bucciarelli-Ducci. NIHR Bristol Cardiovascular Biomedical Research Unit, Bristol Heart Institute, Bristol, Avon, UK; *Presenting Author

\subsection{6/heartjnl-2016-309890.129}

Background Cardiac Magnetic Resonance (CMR) is a valuable tool in the assessment of both ischaemic and non-ischaemic heart disease. The use of CMR in chronic cardiac conditions has already been demonstrated. However, evidence of the impact of CMR on the clinical management on the acute phase of hospital care, is scarce. We sought to evaluate the impact of CMR on diagnosis and clinical decision-making in acute hospitalised patients.

Methods We looked at the 1 year registry data of 2481 consecutive scans (Jan 2014-Dec2014) at a large tertiary cardiothoracic center and identified 283 patients refered for inpatient CMR scan. CMR protocol included short axis and long axis cines, T2 weighted oedema sequences, early and late gadolinium enhancement (LGE) images. Definitions for "significant clinical impact" of CMR were pre-defined and data was collected from clinical records. Categories of significant clinical impact included change in pre-CMR diagnosis, influence on hospitalization period, change in medication, as well as influence on invasive medical procedures such as CABG, angiography and ICD implantation.

Results Of the 283 patients, $8(2.8 \%)$ were excluded due to poor image quality and/or incomplete scans, leaving a sample of 275 patients $(66 \%$ male, mean age 59yrs) with mean ejection fraction of $46 \%+-19$.

Overall, CMR had a significant clinical impact on $68 \%$ of the patients. This included a completely new diagnosis in $27 \%$ of the patients, change in management in $31 \%$ and a total of $10 \%$ of patients had both a new diagnosis and a change in management (see Figure 2). CMR results led to invasive procedures on $27 \%$, avoided invasive procedures on $16 \%$, and had an influence on hospital discharge on $15 \%$ of the patients. $84 \%$ of the patients had echocardiography prior to CMR. CMR confirmed the echo diagnosis in $11 \%$, complemented the echo findings by additing significant new information in $41 \%$ and changed the diagnosis made on echo in $30 \%$ of the cases.

In a multivariable model that included clinical and imaging parameters, age and presence of LGE were the only independent predictor of "significant clinical impact" (LGE p-value. 007, OR 2.782, CI 1.328-5.828) (see Figure 1).

Conclusions CMR had a significant clinical impact on both management and diagnosis in $68 \%$ of acutely hospitalised patients. The presence of LGE was the best independent predictor of significant clinical impact following CMR. 


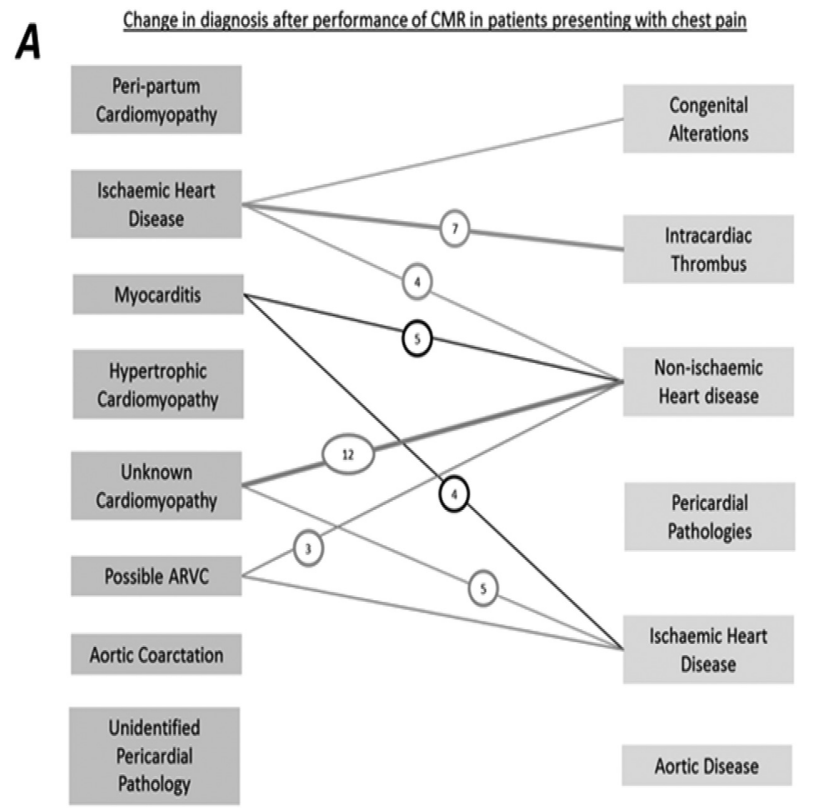

B

Change in diagnosis after performance of CMR in patients presenting with shortness of breath

C

Change in diagnosis after performance of CMR in patients presenting arrhythmias/0OHCA/Syncope

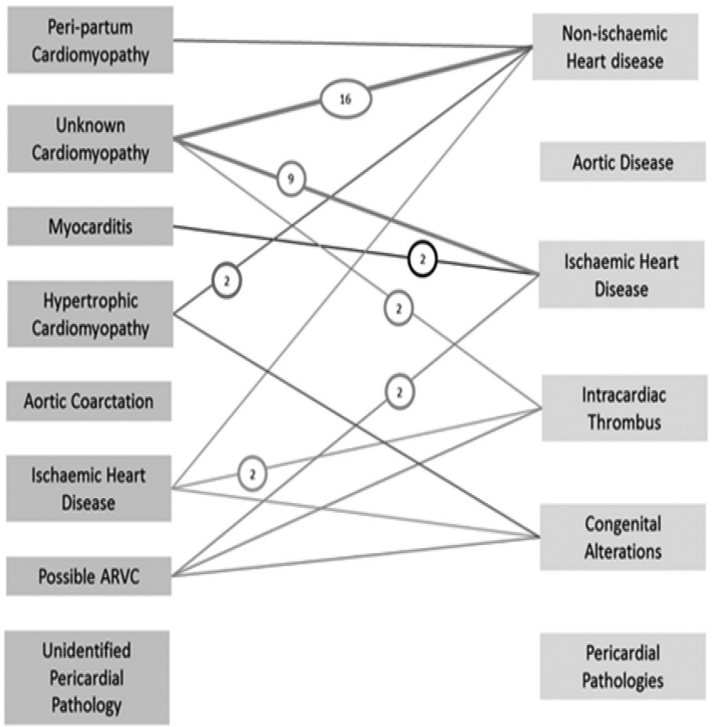

D
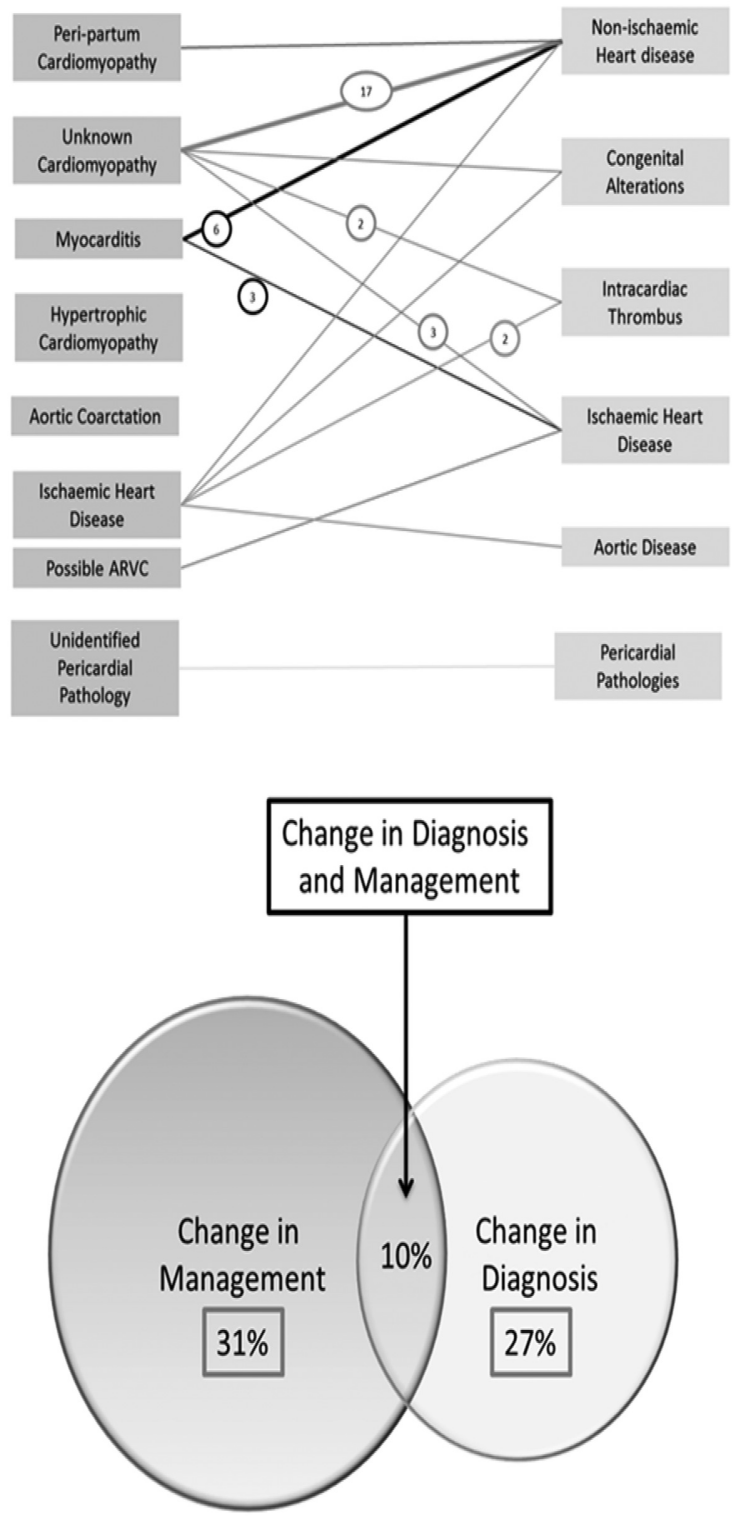

Abstract 129 Figure 1 (A) Change in diagnosis after CMR in patients with chest pain; (B) Change in diagnosis after CMR in patients with shortness of breath; (C) Change in diagnosis after CMR in patients with syncope, arrhythmias and out of hospital cardiac arrest

\section{AN INNOVATIVE AMBULATORY ECHOCARDIOGRAPHY SERVICE PROVIDES RAPID ACCESS TO TESTS, REDUCES LENGTH OF HOSPITAL STAY AND IMPROVES PATIENT CARE}

Alexandra Nowbar*, Francesca Birkinshaw, Ben Goodman, Mehdi Lesko, Gothandaraman Balaji. Northwick Park Hospital; *Presenting Author

\subsection{6/heartjnl-2016-309890.130}

Introduction Demand for urgent Echocardiography in NHS Hospitals is increasing. ${ }^{1}$ Ambulatory care unit provides rapid access to investigations and improved quality of patient care in an outpatient setting. We recently introduced Echocardiography service in Ambulatory Emergency Care Unit (AECU) at Northwick Park Hospital for low-risk cardiac patients

\begin{tabular}{lllll}
\multicolumn{6}{l}{ Abstract 129 Table 1} \\
& Sig. & Odds Ratio & $95 \%$ Conf. Interval & \\
\hline & & & Lower & Upper \\
\hline Sex & .486 & .766 & .361 & 1.622 \\
Age & .028 & 1.026 & 1.003 & 1.050 \\
Troponin & .469 & 1.000 & 1.000 & 1.000 \\
LVEF & .945 & .999 & .972 & 1.027 \\
iEDV & .827 & 1.001 & .989 & 1.014 \\
RWMA & .053 & 2.440 & .987 & 6.033 \\
LGE & .007 & 2.782 & 1.328 & 5.828 \\
Oedema & .672 & .904 & .566 & 1.444 \\
\hline Variable (s): Sex, Age, Troponin, iEDV, RWMA, LGE, Oedema &
\end{tabular}

